\title{
Elevated Expression of CAVI is Associated with Unfavorable Prognosis of Patients with Breast Cancer Who Undergo Surgery and Neoadjuvant Chemotherapy
}

This article was published in the following Dove Press journal:

Cancer Management and Research

\author{
Jia-Hui Ye (D) \\ Jia-Jun Shi $\mathbb{D}^{\prime}$ \\ Xi Yin' \\ Hong-Yan $W_{u^{2}}$ \\ Xin-Yun Xu (D) ${ }^{2}$ \\ Yong-Zhong Yao' \\ Wei-jie Zhang'
}

'General Surgery, Nanjing Drum Tower Hospital, The Affiliated Hospital of Nanjing University Medical School, Nanjing, Jiangsu, People's Republic of China; ${ }^{2}$ Pathology Department, Nanjing Drum Tower Hospital, The Affiliated Hospital of Nanjing University Medical School, Nanjing, Jiangsu, People's Republic of China
Correspondence: Wei-Jie Zhang; YongZhong Yao

No. 32 I Zhonshan Road, Nanjing, Jiangsu,

People's Republic of China

Tel +86 I377077397।

Fax +86 25-83105896

Email zhangweijiel616@l63.com;

loyall006@hotmail.com
Introduction: Neoadjuvant chemotherapy (NACT), which is standard treatment for locally advanced breast cancer, improves the resectability of patients with early breast cancer and reduces the extent of breast and axillary surgery. Caveolin-1 (CAV1) is implicated in human cancers, although its utility for cancer prognosis is unknown. Here, we investigated the expression of CAV1 in breast cancer tissues to evaluate its prognostic significance on patients with breast cancer administered NACT.

Methods: CAV1 expression in 80 breast cancer tissue samples was evaluated using immunohistochemistry (IHC). The association between CAV1 levels and clinical factors was analyzed using the chi-square test and that between CAV1 and prognosis was evaluated using multivariate Cox regression and Kaplan-Meier analyses.

Results: High levels of CAV1 were significantly associated with survival, and patients with overexpression of CAV1 had a poor prognosis. Adjusted multivariate Cox regression analyses revealed that a high level of CAV1 expression was an independent, significant prognostic factor for patients with breast cancer treated with NACT.

Discussion: Overexpression of CAV1 in patients with breast cancer administered NACT was associated with shorter disease-free survival and overall survival. Therefore, high levels of CAV1 may serve as a prognostic biomarker for such patients.

Keywords: CAV1, IHC, clinical outcome, biomarker

\section{Introduction}

Breast cancer is the most common malignancy affecting women worldwide. In 2018, approximately 2.1 million women were diagnosed with breast cancer and 627,000 died. $^{1}$ Despite advanced chemotherapy and targeted therapy, most patients with breast cancer are at risk tumor for recurrence and metastasis.

The caveolin family comprises caveolin-1, caveolin-2, and caveolin-3 (CAV13 ), which are expressed on the cell membrane. CAV1, which is a major structural protein of caveolae, mediates tumorigenesis and tumor progression. Numerous cell types express CAV1, such as smooth muscle cells, endothelial cells, skeletal myoblasts, and fibroblasts. ${ }^{2,3}$ Moreover, CAV1 mediates multiple physiological processes including membrane trafficking, cholesterol homeostasis, signal transduction, and tumor progression., 
CAV1 was first detected in an attempt to identify new cytoskeletal muscles, and evidence indicates that CAV1 contributes to malignant transformation, promotes the migration of cancer cells, and confers a metastatic phenotype. Further, CAV1 contributes to tumor proliferation, survival, invasion, apoptosis, autophagy, and resistance to chemotherapy. ${ }^{6}$ CAV1 is therefore considered as a potential therapeutic target for cancers.

In contrast, other studies show that CAV1 acts as a tumor suppressor, ${ }^{7,8}$ despite evidence cited above identifying it as an oncoprotein in certain cancers. For example, the expression of CAV1 in pancreatic ductal adenocarcinoma is significantly associated with tumor size, grade, and stage ${ }^{9}$ and convincing evidence shows that patients' renal cancer with elevated levels of CAV1 expression experience shorter overall survival (OS). ${ }^{10}$

CAV1 may contribute to the progression and metastasis of hepatocellular carcinoma (HCC) through the inhibition of autophagy or by inducing the epithelial-mesenchymal transition via the Wnt/b-catenin signal transduction pathway. ${ }^{11} \mathrm{CAV} 1$ is a negative predictor of the prognosis of lung cancer6. In brain tumors (eg, oligodendrogliomas, ependymomas, meningiomas, vestibular schwannomas, and brain metastases), CAV1 may influence the molecular mechanisms underlying pathobiology and may predict tumor grade, recurrence, and prognosis. ${ }^{12}$

During the progression of prostate cancer, loss of CAV1 from the microenvironment leads to the metastasis of tumor cells through a mechanism involving TGF- $\beta 1$ and SNGC signaling through the AKT signal transduction pathway. ${ }^{13}$ Further, evidence indicates that loss of CAV1 expression serves as the most significant prognostic factor of OS of patients with triple-negative (TNBC) and basal-like breast cancers. ${ }^{14}$

However, the clinical utility of CAV1 as a prognostic marker for patients who undergo NACT is unknown. Therefore, a better understanding of CAV1 expression in patients with breast cancer administered NACT may improve treatment strategies and assessment of a patient's outcome. For this purpose, here we evaluated the role of CAV1 expression in disease-free survival (DFS) and OS of such patients.

\section{Patients and Methods}

We analyzed tissues collected from 80 patients with breast cancer who underwent NACT and subsequent surgery at Nanjing Drum Towel Hospital between 2010 and 2019. The study was approved by the Ethics Committees of Nanjing Drum Towel Hospital and the Affiliated Hospital of Nanjing University Medical School (2020-134) and adhered to the tenets of the Declaration of Helsinki. This was a retrospective study. The complete medical records and follow-up data of these patients were available. The inclusion criterion for this study was women with breast cancer treated with NACT at the same hospital. These patients underwent subsequent surgery when NACT was completed, and they were excluded if they did not undergo breast surgery or finish chemotherapy before surgery. Data on age, tumor size, diagnosis, and length of surgery were collected from patients' medical records. Follow-up information was obtained by phone investigations. The patients' ages at surgery ranged from 27 to 74 years (mean, 54 years). The date of the last follow-up was December 21, 2019. Patients' detailed clinical and pathological information are shown in Table 1 .

Table I Correlation Analysis of CAVI Expression with Clinicopathological Characteristics of 80 Breast Carcinomas

\begin{tabular}{|c|c|c|c|}
\hline & \multicolumn{2}{|l|}{ CAVI } & \multirow[t]{2}{*}{$P$ value } \\
\hline & $\begin{array}{l}\text { Low } \\
\text { Expression }\end{array}$ & $\begin{array}{l}\text { High } \\
\text { Expression }\end{array}$ & \\
\hline \multicolumn{4}{|l|}{ Age (y) } \\
\hline$\geq 50$ & 27 (33.75\%) & 21 (40\%) & 0.402 \\
\hline$<50$ & 21 (26.25\%) & II (13.75\%) & \\
\hline \multicolumn{4}{|l|}{$\begin{array}{l}\text { Tumor size } \\
(\mathrm{cm})\end{array}$} \\
\hline$>2 \mathrm{~cm}$ & 28 (35\%) & 15 (I8.75\%) & 0.059 \\
\hline$\leq 2 \mathrm{~cm}$ & 7 (8.75\%) & II (13.75\%) & \\
\hline \multicolumn{4}{|l|}{$\mathbf{N}$ classification } \\
\hline 0 & 14 (I7.5\%) & II (13.75\%) & $0.76 \mathrm{I}$ \\
\hline I & 10 (I2.5\%) & 5 (6.25\%) & \\
\hline 2 & 7 (8.75\%) & $5(6.25 \%)$ & \\
\hline 3 & $4(5 \%)$ & $5(5 \%)$ & \\
\hline \multicolumn{4}{|l|}{ ER status } \\
\hline Positive & $28(35 \%)$ & II (13.75\%) & 0.400 \\
\hline Negative & $20(25 \%)$ & $10(12.5 \%)$ & \\
\hline \multicolumn{4}{|l|}{ PR status } \\
\hline Positive & 26 (32.5\%) & 19 (23.75\%) & 0.532 \\
\hline Negative & 22 (27.5\%) & 12 (I5\%) & \\
\hline \multicolumn{4}{|l|}{ ERBB2 status } \\
\hline $3+$ & $20(25 \%)$ & $16(20 \%)$ & 0.845 \\
\hline $2+$ & $12(15 \%)$ & $8(10 \%)$ & \\
\hline $1+$ & 7 (8.75\%) & 3 (3.75\%) & \\
\hline 0 & 9 (II.25\%) & $5(6.25 \%)$ & \\
\hline \multicolumn{4}{|l|}{ Survival status } \\
\hline Yes & 45 (56.25\%) & $24(30 \%)$ & 0.017 \\
\hline Not & $3(3.75 \%)$ & $8(10 \%)$ & \\
\hline
\end{tabular}


Histopathology and immunohistochemistry findings were evaluated by pathologists and assigned $\mathrm{H}$-scores (see below).

\section{Immunohistochemical Analysis}

Representative sections (3- $\mu \mathrm{m}$ thick) from breast cancer tissues were deparaffinized in xylene and rehydrated in graded concentrations of ethanol. Antigen retrieval was achieved by boiling sections for $3 \mathrm{~min}$ in retrieval buffer (ethylene diamine tetraacetic acid, $\mathrm{pH}$ 8.0). Phosphate-buffered saline (PBS) containing 1\% Tween 20 was used to wash the sections three times. Next, sections were incubated in $3 \% \mathrm{H}_{2} \mathrm{O}_{2}$ for 15 min to inhibit endogenous tissue peroxidase activity. After washing with PBS three times, the slides were incubated with a mouse anti-CAV1 antibody (ZM-0050, Zhongshan Golden Bridge Bio-technology, Beijing, China) overnight at $4^{\circ} \mathrm{C}$. Secondary antibodies and diaminobenzidine were employed to detect immune complexes. All stained slides were observed and evaluated using a visible-light microscope.

The intensity of CAV1 staining was scored as 0 , undetectable; 1 , weak; 2, moderate; and 3 , intense, according to the $\mathrm{H}$-scoring method. The $\mathrm{H}$-scoring method is based on the total number of tumor stromal tissues expressing CAV1 and the intensity of staining. The H-score is calculated as follows: score $=$ percentage stained tumor stromal tissue $\times$ staining intensity. For assessment of CAV1 expression, H-scores higher than average were considered high expression and $\mathrm{H}$-scores between 0 and the average were considered low expression.

\section{Statistical Analysis}

Statistical analyses were performed using the Statistical package for Social Science 26 (26.0; IBM Corp., Armonk, NY, USA). GraphPad Prism (8.0, GraphPad Software Inc. San Diego, CA, USA) was used as well. We used the chi-square test to investigate the significance of the associations between patients' variables such as age, tumor size, N classifications, ER status, PR status, ERBB2 status, and survival status. Cox regression analysis was performed to evaluate the significance of the influence of prognostic factors on survival in a multivariate context (adjusting for confounding variables). Two-tailed $\mathrm{p}<0.05$ indicates a significant difference.

\section{Results}

\section{Association of CAVI Expression Levels with Clinicopathological Variables}

The relationship between CAV1 and clinicopathological variables of 80 patients with breast cancer is shown in
Table 1. There was a significant relationship $(p=0.017)$ between CAV1 expression and the prognosis of breast cancer patients who were administered NACT (Table 1). There was no significant correlation with age $(\mathrm{p}=0.402)$, tumor size $(p=0.059), N$ classification $(p=0.761)$, ER status $(\mathrm{p}=0.400), \quad$ PR status $(\mathrm{p}=0.532)$, or ERBB2 status $(\mathrm{p}=0.845)$.

\section{Expression of CAVI in Breast Tumor Tissue}

CAV1 is expressed by different cell types such as fat cells, fibroblasts, endothelial cells, smooth muscle cells, and breast epithelial cells. ${ }^{10}$ Immunohistochemistry (IHC) was used to analyze CAV1 expression (Figure 1A and B). CAV1 was expressed in the tumor stroma of all breast cancer samples and was abundant and predominantly localized in breast

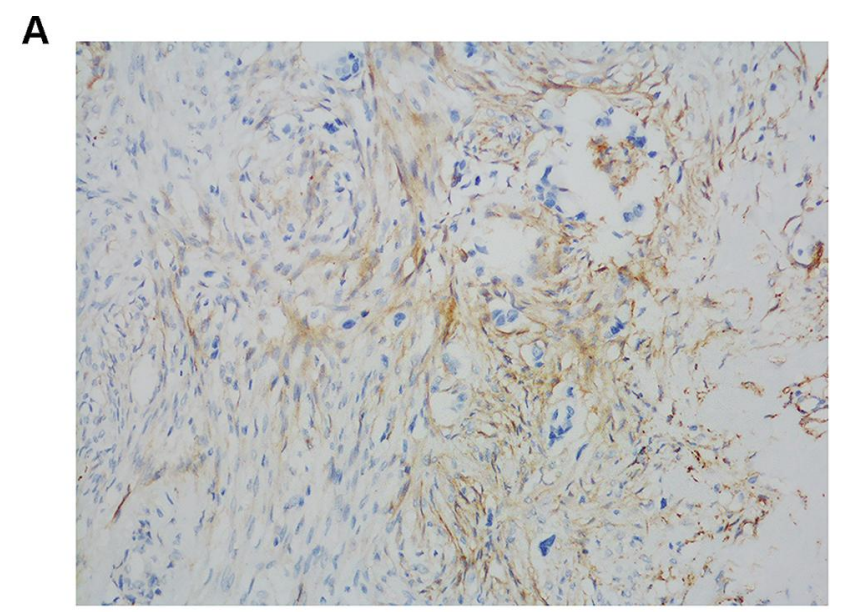

$\mathbf{B}$

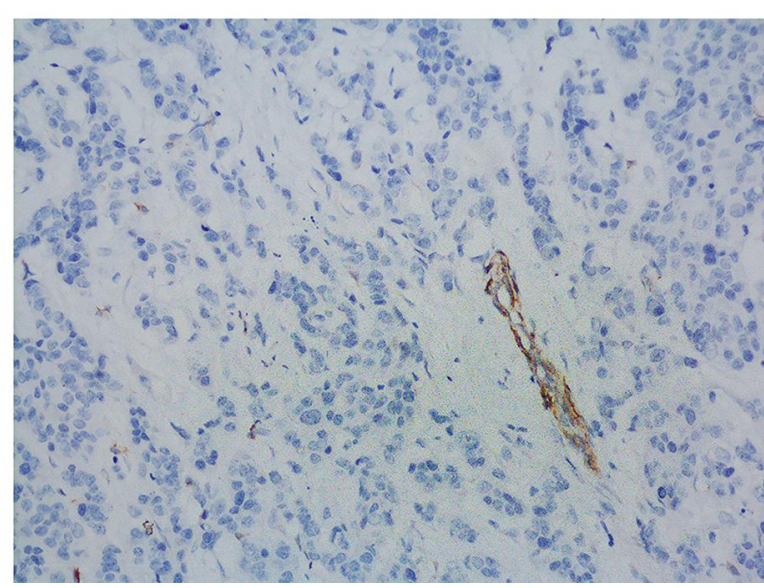

Figure I CAVI expression in NACT-breast cancer patients. Tissue section cut from formalin-fixed paraffin-embedded NACT breast cancer samples were immunostained with antibody directed against cav-I (A and B). Note the presence of Cav-I immunostaining in tumor tissue in (A), and the absence of Cav-I immunostaining in tumor-adjacent tissue in (B). Scale Bar:200 $\mu \mathrm{m}$ for (A and B). The brown staining in (A) represented stroma. And the blue staining in the rest area represented cancer cells. 
cancer tissues. The staining intensities and patterns of normal tissues adjacent to the tumor tissues were used as baseline. Representative examples of CAV1 immunostaining are shown in Figure 1A. CAV1 expression was undetectable in nontumor tissues (Figure 1B). Quantification of CAV1 expression was low in $60 \%$ of tumor samples and high in $40 \%$.

\section{Prognostic Value of CAVI IHC for Predicting Clinical Outcomes in Breast Cancer Patients Administered NACT}

We next used IHC to investigate the value of CAV1 as a prognostic biomarker of the clinical outcomes of patients with breast cancer who were administered NACT. Clinical data were collected from hospital records and telephone interviews. When this manuscript was submitted for publication, $11(13.75 \%)$ were deceased and 69 (86.25\%) were alive.

The DFS of patients with high CAV1 expression was significantly shorter compared with those with low CAV1 expression ( $p=0.0042$ ) (Figure 2A). Univariate Cox's regression analysis revealed that high expression of CAV1 significantly correlated with shorter DFS $(p=0.011$, hazard ratio $[\mathrm{HR}]=5.648)$. In contrast, there was no significant association of DFS with age $(\mathrm{p}=0.079, \mathrm{HR}=6.330)$, tumor size $(\mathrm{p}=0.782, \mathrm{HR}=0.822)$, ER status $(\mathrm{p}=0.550, \mathrm{HR}=0.697), \mathrm{PR}$ status $(p=0.152, H R=0.407)$, or ERBB2 status $(p=0.642$ $\mathrm{HR}=1.331)$. There was a statistically significant association ( $\mathrm{p}=0.011, \mathrm{HR}=5.666$ ) of CAV1 expression with OS (Figure 2B). The results of univariate analysis of DFS and OS are summarized in Tables 2 and 3.

Multivariate Cox regression analysis showed a significant effect of CAV1 on DFS and OS, but not with other factors, except $\mathrm{N}$ classification $(\mathrm{p}=0.016$ and 0.029 , respectively). Lymph node metastasis serves as a prognostic marker for patients with breast cancer. Thus, CAV1 expression levels and N classification were significant factors for predicting DFS and OS of our patient population (Tables 4 and 5). These data support the conclusion that CAV1 independently influenced prognosis.

\section{Discussion}

Here we used IHC to investigate the clinical significance of CAV1 expression levels of patients with breast cancer administered NACT. CAV1 antagonistically regulates cellular processes related to inflammation. ${ }^{15}$ Our result indicates the relevance of CAV1 for predicting the prognosis of such patients and that the analysis of CAV1 expression may therefore contribute to the understanding of the

\section{A Disease-free survival $(n=80)$}

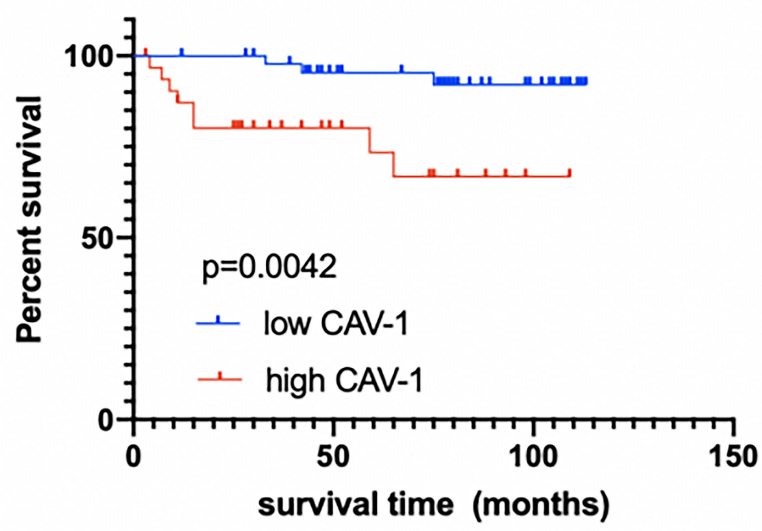

B

overall survival $(n=80)$

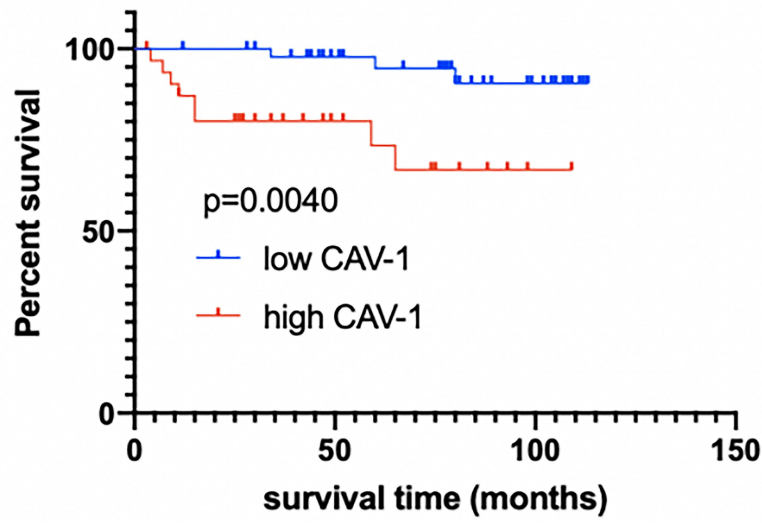

Figure 2 Kaplan-Meier analysis showing the predictive value of CAVI in NACTbreast cancer patients with low or high CAVI expression. (A) Comparison of disease-free survival (DFS) based on the expression levels of CAVI. (B) Comparison of overall survival (OS) as a function of the expression levels of CAVI. The blue line indicated low expression of CAVI and the red line indicated high expression of CAVI.

influence of CAV1 on breast cancer. Moreover, our present data provide support for the conclusion that CAV1 shows promise as a biomarker for predicting the prognosis of such patients.

Table 2 Univariate Analysis of DFS According to Clinicopathological Factors

\begin{tabular}{|c|c|c|c|}
\hline & OR & $95 \% \mathrm{Cl}$ & $\mathbf{P}$ \\
\hline Age ( $\geq 50$ years vs $<50$ years) & 6.330 & $0.810-49.47 \mid$ & 0.079 \\
\hline Tumor size $(>2 \mathrm{~cm}$ vs $\leq 2 \mathrm{~cm})$ & 0.822 & $0.205-3.291$ & 0.782 \\
\hline $\mathrm{N}$ classification $(0$ vs $1,2,3)$ & 7.112 & $0.0883-57.255$ & 0.065 \\
\hline ER status (positive vs negative) & 0.697 & $0.213-2.283$ & 0.550 \\
\hline PR status (positive vs negative) & 0.407 & $0.119-1.1391$ & 0.152 \\
\hline $\operatorname{ERBB} 2(3+$ vs $0,1+, 2+)$ & 1.331 & $0.399-4.445$ & 0.642 \\
\hline CAVI (high vs low) & 5.648 & I.487-2I.457 & 0.011 \\
\hline
\end{tabular}


Table 3 Univariate Analysis of OS According to Clinicopathological Factors

\begin{tabular}{|c|c|c|c|}
\hline & OR & $95 \% \mathrm{Cl}$ & $P$ \\
\hline Age ( $\geq 50$ years vs $<50$ years) & 5.903 & $0.754-46.204$ & 0.091 \\
\hline Tumor size $(>2 \mathrm{~cm}$ vs $\leq 2 \mathrm{~cm})$ & 0.850 & $0.212-3.407$ & 0.819 \\
\hline $\mathrm{N}$ classification (0 vs $1,2,3)$ & $6.67 \mid$ & $0.828-53.759$ & 0.075 \\
\hline ER status (positive vs negative) & 0.654 & $0.199-2.145$ & 0.484 \\
\hline PR status (positive vs negative) & 0.389 & $0.114-1.329$ & 0.132 \\
\hline ERBB2 $(3+$ vs $0,1+, 2+)$ & 1.457 & $0.436-4.866$ & 0.541 \\
\hline CAVI (high vs low) & 5.666 & $1.482-21.663$ & 0.011 \\
\hline
\end{tabular}

Table 4 Multivariate Cox Proportional Hazard Analysis of DFS of Patients with Breast Cancer Administered NACT

\begin{tabular}{|l|l|l|l|}
\hline & OR & $\mathbf{9 5 \%}$ CI & $P$ \\
\hline Age ( $\geq 5$ yyears vs $<50$ years) & 4.634 & $0.528-40.697$ & 0.167 \\
Tumor size (>2cm vs $\leq 2 \mathrm{~cm})$ & 0.396 & $0.032-4.855$ & 0.469 \\
N classification (0 vs I,2,3) & 37.893 & $1.946-737.961$ & 0.016 \\
ER status (positive vs negative) & 0.574 & $0.054-6.129$ & 0.646 \\
PR status (positive vs negative) & 0.148 & $0.009-2.339$ & 0.175 \\
ERBB2 (3+ vs 0,I+,2+) & 0.563 & $0.099-3.193$ & 0.517 \\
CAVI (high vs low) & 15.620 & $1.778-137.242$ & 0.013 \\
\hline
\end{tabular}

Table 5 Multivariate Cox Proportional Hazard Analysis of OS of Patients with Breast Cancer Administered NACT

\begin{tabular}{|l|l|l|l|}
\hline & OR & $\mathbf{9 5 \%} \mathbf{C l}$ & $\mathbf{P}$ \\
\hline Age $(\geq 5$ yyears vs $<50$ years) & 2.676 & $0.308-23.227$ & 0.372 \\
Tumor size $(>2 \mathrm{~cm}$ vs $\leq 2 \mathrm{~cm})$ & 0.369 & $0.030-4.509$ & 0.435 \\
$\mathrm{~N}$ classification $(0$ vs I,2,3) & 27.483 & $1.401-539.054$ & 0.029 \\
ER status (positive vs negative) & $0.4 I I$ & $0.039-4.288$ & 0.458 \\
PR status (positive vs negative) & 0.201 & $0.014-2.862$ & 0.236 \\
ERBB2 (3+ vs 0,I+,2+) & 1.473 & $0.338-6.415$ & 0.606 \\
CAVI (high vs low) & 8.527 & $1.253-58.019$ & 0.028 \\
\hline
\end{tabular}

Consistent with our findings presented here, other evidence indicates that CAV1 may serve as biomarker for the response to albumin-based cancer therapeutic drugs. ${ }^{16}$ For example, in HCC arising from nonalcoholic fatty liver disease, CAV1 overexpression contributes to the survival of HCC cells in fatty acid-rich environments and therefore may serve as a target of therapy. ${ }^{17}$

CAV1 is overexpressed in metastatic prostate cancer, and its high expression predicts recurrence after radical prostatectomy. Further, serum levels of CAV1 differentiate patients with prostate cancer from those with benign prostatic hyperplasia. ${ }^{18}$ Moreover, $C A V 1$ is considered a target gene, because its product promotes the chemoresistance of ovarian cancer through the NOTCH1/AKT/NFKB1 signal transduction pathway. ${ }^{19}$
In the present study, differential CAV1 expression was significantly associated with DFS and OS. We further found that a high CAV1 level serves as a prognostic factor for patients with breast cancer administered NACT who underwent radical surgery. Moreover, the $\mathrm{N}$ classification was significantly associated with shorter survival of patients with breast cancer.

A previous study investigated the relationship between high CAV1 expression and poor prognosis of breast cancer. ${ }^{20}$ Further, CAV1 levels in breast cancerassociated fibroblasts are associated with poor prognosis, ${ }^{21}$ and the absence of CAV1 expression in the stroma is associated with poor prognosis of patients with breast cancer, although CAV1 and CAV2 expression in tumor epithelial cells is not. ${ }^{22}$ Moreover, CAV1 is considered a therapeutic biomarker of the effects of breast cancer treatment, and epithelial and stromal CAV1 levels predict the prognosis of patients with breast cancer.

The results of the present study are consistent with findings that high expression of CAV1 in invasive breast cancer cells partially affects tumor aggressiveness and predicts poor prognosis. ${ }^{23}$ Similarly, here we show that CAV1 expression was an independent risk factor for poor survival. Further, we identified CAV1 as a new predictive biomarker for patients with breast cancer administered NACT and that low CAV1 levels were associated with longer survival.

When we analyzed CAV1 expression in patients with or without TNBC, we found that CAV1 levels were not significantly associated with these molecular subtypes (Table 6).

In conclusion, we show here, for the first time to our knowledge, that high CAV1 levels were significantly associated with poor prognosis of patients administered NACT who undergo radical breast cancer surgery. To validate these results, further assessment is required to reveal an association between CAV1 and signal transduction pathways, which will likely identify more treatment options useful for targeting CAV1 before and after surgery.

Table 6 Correlation Analysis of CAVI Expression with Molecular Subtypes of Breast Cancer

\begin{tabular}{|l|l|l|l|}
\hline $\begin{array}{l}\text { Molecular } \\
\text { Subtypes }\end{array}$ & $\begin{array}{l}\text { Low CAVI } \\
(\mathbf{n = 4 8 )}\end{array}$ & $\begin{array}{l}\text { High CAVI } \\
(\mathbf{n = 3 2})\end{array}$ & $\boldsymbol{P}$ \\
\hline $\begin{array}{l}\text { TNBC } \\
\text { Without TNBC }\end{array}$ & $\begin{array}{l}5(6.25 \%) \\
43(53.75 \%)\end{array}$ & $\begin{array}{l}\text { I }(1.25 \%) \\
31(38.75 \%)\end{array}$ & 0.225 \\
\hline
\end{tabular}




\section{Disclosure}

The authors report no conflicts of interest in this work.

\section{References}

1. Bray F, Ferlay J, Soerjomataram I, et al. Global cancer statistics 2018: GLOBOCAN estimates of incidence and mortality worldwide for 36 cancers in 185 countries. CA Cancer J Clin. 2018;68 (6):394-424. doi:10.3322/caac.21492

2. Huang Q, Zhong W, Hu Z, et al. A review of the role of CAV1 in neuropathology and neural recovery after ischemic stroke. J Neuroinflammation. 2018;15(1):348. doi:10.1186/s12974-018-1387-y

3. Anderson RG. The caveolae membrane system. Annu Rev Biochem. 1998;67(1):199-225. doi:10.1146/annurev.biochem.67.1.199

4. Wang S, Wang N, Zheng Y, et al. Caveolin-1: an oxidative stress-related target for cancer prevention. Oxid Med Cell Longev. 2017;2017:7454031. doi:10.1155/2017/7454031

5. Nguyen KC, Cho KA. Versatile functions of caveolin-1 in aging-related diseases. Chonnam Med J. 2017;53(1):28-36.

6. Shi YB, Li J, Lai XN, et al. Multifaceted roles of caveolin-1 in lung cancer: a new investigation focused on tumor occurrence, development and therapy. Cancers (Basel). 2020;12(2):E291. doi:10.3390/ cancers12020291

7. Wu KN, Queenan M, Brody JR, et al. Loss of stromal caveolin-1 expression in malignant melanoma metastases predicts poor survival. Cell Cycle. 2011;10(24):4250-4255. doi:10.4161/cc.10.24.18551

8. Zhao Z, Han FH, Yang SB, et al. Loss of stromal caveolin-1 expression in colorectal cancer predicts poor survival. World J Gastroenterol. 2015;21(4):1140-1147. doi:10.3748/wjg.v21.i4.1140

9. Tanase CP, Dima S, Mihai M, et al. Caveolin-1 overexpression correlates with tumour progression markers in pancreatic ductal adenocarcinoma. J Mol Histol. 2009;40(1):23-29. doi:10.1007/ s10735-008-9209-7

10. Campbell L, Al-Jayyoussi G, Gutteridge R, et al. Caveolin-1 in renal cell carcinoma promotes tumour cell invasion, and in co-operation with pERK predicts metastases in patients with clinically confined disease. J Transl Med. 2013;11(1):255. doi:10.1186/1479-5876-11255

11. Liu WR, Jin L, Tian MX, et al. Caveolin-1 promotes tumor growth and metastasis via autophagy inhibition in hepatocellular carcinoma. Clin Res Hepatol Gastroenterol. 2016;40(2):169-178. doi:10.1016/j. clinre.2015.06.017
12. Ocak PE, Ocak U, Tang J, et al. The role of caveolin-1 in tumors of the brain - functional and clinical implications. Cell Oncol (Dordr). 2019;42(4):423-447.

13. Ayala G, Morello M, Frolov A, et al. Loss of caveolin-1 in prostate cancer stroma correlates with reduced relapse-free survival and is functionally relevant to tumour progression. J Pathol. 2013;231 (1):77-87. doi:10.1002/path.4217

14. Witkiewicz AK, Dasgupta A, Sammons S, et al. Loss of stromal caveolin-1 expression predicts poor clinical outcome in triple negative and basal-like breast cancers. Cancer Biol Ther. 2010;10 (2):135-143. doi:10.4161/cbt.10.2.11983

15. de Almeida CJ. Caveolin-1 and caveolin-2 can be antagonistic partners in inflammation and beyond. Front Immunol. 2017;8:1530. doi:10.3389/fimmu.2017.01530

16. Chatterjee M, Ben-Josef E, Robb R, et al. Caveolae-mediated endocytosis is critical for albumin cellular uptake and response to albumin-bound chemotherapy. Cancer Res. 2017;77(21):5925-5937. doi:10.1158/0008-5472.CAN-17-0604

17. Takeda M, Sakaguchi T, Hiraide T, et al. Role of caveolin-1 in hepatocellular carcinoma arising from non-alcoholic fatty liver disease. Cancer Sci. 2018;109(8):2401-2411. doi:10.1111/cas.13659

18. Tahir SA, Ren C, Timme TL, et al. Development of an immunoassay for serum caveolin-1: A novel biomarker for prostate cancer. Clin Cancer Res. 2003;9(10 Pt 1):3653-3659.

19. Zou W, Ma X, Hua W, et al. Caveolin-1 mediates chemoresistance in cisplatin-resistant ovarian cancer cells by targeting apoptosis through the Notch-1/Akt/NF-kB pathway. Oncol Rep. 2015;34(6):3256-3263. doi:10.3892/or.2015.4320

20. Qian N, Ueno T, Kawaguchi-Sakita N, et al. Prognostic significance of tumor/stromal caveolin-1 expression in breast cancer patients. Cancer Sci. 2011;102(8):1590-1596. doi:10.1111/j.1349-7006.2011. 01985.x

21. Samantha AS, Andrew MH, Deborah LH, et al. Clinical and functional significance of loss of caveolin-1 expression in breast cancer-associated fibroblasts. J Pathol. 2012;227(4):490-498. doi:10. 1002/path.4034

22. Ma X, Liu L, Nie W, et al. Prognostic role of caveolin in breast cancer: a meta-analysis. Breast. 2013;22(4):462-469. doi:10.1016/j. breast.2013.03.005

23. Qian XL, Pan YH, Huang QY, et al. Caveolin-1: a multifaceted driver of breast cancer progression and its application in clinical treatment. Onco Targets Ther. 2019;12:1539-1552. doi:10.2147/OTT.S191317

\section{Publish your work in this journal}

Cancer Management and Research is an international, peer-reviewed open access journal focusing on cancer research and the optimal use of preventative and integrated treatment interventions to achieve improved outcomes, enhanced survival and quality of life for the cancer patient.
The manuscript management system is completely online and includes a very quick and fair peer-review system, which is all easy to use. Visit http://www.dovepress.com/testimonials.php to read real quotes from published authors. 